\title{
Abundances of planetary nebula NGC 2392 ${ }^{\star}$
}

\author{
S. R. Pottasch ${ }^{1}$, J. Bernard-Salas ${ }^{2}$, and T. L. Roellig ${ }^{3}$ \\ 1 Kapteyn Astronomical Institute, PO Box 800, 9700 AV Groningen, The Netherlands \\ e-mail: pottasch@astro.rug.nl \\ 2 Center for Radiophysics and Space Research, Cornell University, Ithaca, NY 14853, USA \\ 3 NASA Ames Research Center, MS 245-6, Moffett Field, CA 94035-1000, USA
}

Received 9 November 2007 /Accepted 10 January 2008

\begin{abstract}
The spectra of the planetary nebula NGC 2392 is reanalysed using spectral measurements made in the mid-infrared with the Spitzer Space Telescope. The aim is to determine the chemical composition of this object. We also make use of IUE and ground based spectra. Abundances determined from the mid-infrared lines, which are insensitive to electron temperature, are used as the basis for the determination of the composition, which are found to differ somewhat from earlier results. The abundances found, especially the low value of helium and oxygen, indicate that the central star was originally of rather low mass. Abundances of phosphorus, iron, silicon and chlorine have been determined for the first time in this nebula. The variation of electron temperature in this nebula is very clear, reaching quite high values close to the center. The temperature of the central star is discussed in the light of the observed high stages of ionization. The nebular information indicates that the spectrum of the star deviates considerably from a blackbody.
\end{abstract}

Key words. ISM: planetary nebulae: individual: NGC 2392 - infrared: ISM

\section{Introduction}

NGC 2392 (PN G197.8+17.3) is a bright planetary nebula with a rather high radial velocity, located in the direction of the galactic anticenter. A photograph of the nebula is shown in Fig. 1. The nebula has a bright inner ellipsoidal, almost round shell of about $18^{\prime \prime}$ diameter. This is surrounded by an almost spherical region of considerably lower emission, with a diameter of about $40^{\prime \prime}$. Both the inner and outer regions contain some structure. Analysis of the kinematics of the nebula (O'Dell \& Ball 1985) indicates that the inner region is expanding quite rapidly with respect to the outer region, which is also slowly expanding. The nebula is located 17 degrees above the galactic plane and has only a small extinction. Because of its brightness it is considered a nearby nebula: most of the uncertain distance estimates vary between 1 and $2 \mathrm{kpc}$.

The nebula has a bright central star $(V=10.53)$ which has been studied by several authors. Pauldrach et al. (2004) have compared the stellar spectrum with a model atmosphere and conclude that the star has an effective temperature $T_{\text {eff }}=40000 \mathrm{~K}$. While this is slightly higher than the hydrogen Zanstra temperature of about $36000 \mathrm{~K}$, it is considerably less than the ionized helium Zanstra temperature which is close to $70000 \mathrm{~K}$. Pauldrach et al. (2004) have noted this discrepancy and admit that they are unable to explain the additional He-ionizing photons which are needed to explain the high $T_{z}(\mathrm{HeII})$. Tinkler \& Lamers (2002) prefer to assign a much higher effective temperature to the star based on the high ionized helium Zanstra temperature and the high energy balance temperature: $T_{\text {eff }}=73000 \mathrm{~K}$. It is clear that the excitation in the nebula is much higher than can be accounted

\footnotetext{
^ Based on observations with the Spitzer Space Telescope, which is operated by the Jet Propulsion Laboratory, California Institute of Technology.
}

for by a temperature of $T_{\text {eff }}=40000 \mathrm{~K}$. This was already noted by Natta et al. (1980) who showed that higher temperature ionizing radiation was necessary for all ions found in the nebula: the higher the ionization potential of the ion studied, the higher the ionizing radiation temperature must be. Why this result is not found in the analysis of the stellar spectrum is not understood.

The purpose of this paper is to study the element abundances in this nebula with the help of mid-infrared spectra, in the hope that the chemical abundances will shed some light on the evolution of this nebula-central star combination. Abundances have been studied by Barker (1991) and by Henry et al. (2000). Both of these groups use optical nebular spectra (taken by themselves) and ultraviolet IUE spectra. Barker (1991) has measurements taken at five different positions in the nebula. He uses low dispersion IUE measurements taken with the small aperture ( $3^{\prime \prime}$ diameter). Henry et al. (2000) also take spectra at five different positions in the nebula but they use low dispersion IUE measurements taken with the large aperture $\left(10^{\prime \prime} \times 23^{\prime \prime}\right)$. No abundance changes as a function of the position in the nebula are found by either group. Both groups agree on the abundances of $\mathrm{N}, \mathrm{O}$ and $\mathrm{Ne}$ to within $20 \%$. However their carbon abundances differ by more than a factor of 6 and the helium abundances differ by almost $30 \%$. Henry et al. (2000) do not discuss the abundance of other elements, while Barker also determines sulfur and argon.

We have measured the spectrum of NGC 2392 in the mid-infrared with the IRS spectrograph of the Spitzer Space Telescope (Werner et al. 2004). The use of the mid-infrared spectrum permits a more accurate determination of the abundances. The reasons for this have been discussed in earlier studies (e.g. see Pottasch \& Beintema 1999; Pottasch et al. 2000, 2001; Bernard Salas et al. 2001), and can be summarized as follows.

The most important advantage is that the infrared lines originate from very low energy levels and thus give an abundance 


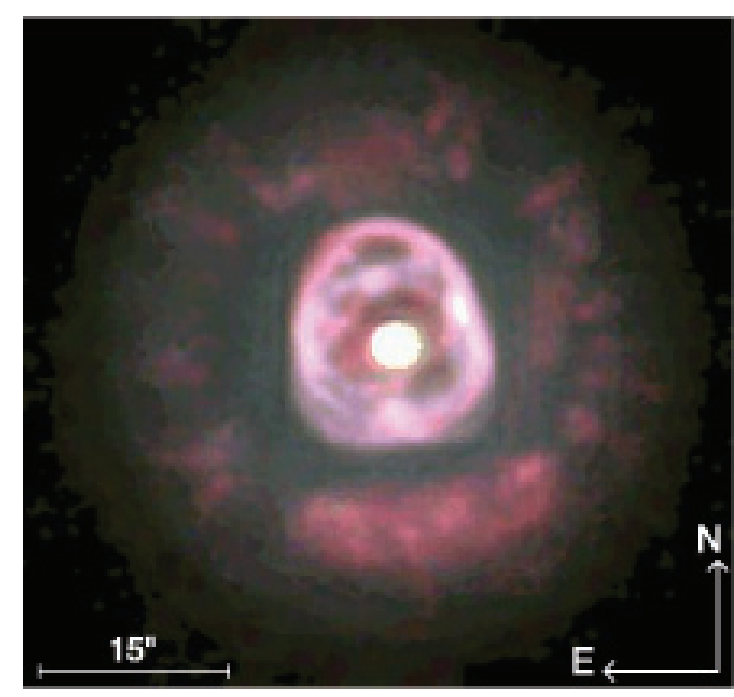

Fig. 1. An HST image of NGC 2392.

which is not sensitive to the temperature in the nebula, nor to possible temperature fluctuations. Furthermore, when a line originating from a high-lying energy level in the same ion is observed, it is possible to determine an average electron temperature at which the lines in that particular ion are formed. When this average temperature for many ions is determined, it is possible to make a plot of electron temperature against ionization potential, which can be used to determine the average electron temperature (or range of temperatures) for which ions of a particular ionization potential are observed. As will be shown, this is particularly important in NGC 2392 for which a large gradient in electron temperature is found. This had not been taken into account in the earlier abundance studies of this nebula.

Use of the infrared spectra have further advantages. One of them is that the number of observed ions used in the abundance analysis is approximately doubled, which removes the need for using large ionization correction factors (ICFs), thus substantially lowering the uncertainty in the abundance. A further advantage is that the extinction in the infrared is almost negligible, eliminating the need to include sometimes large correction factors. This is not very important in this nebula. The number of elements that can be measured increases when including the infrared. In NGC 2392 phosphorus, iron and silicon can be measured in the mid-infrared. This nebula has not been measured by $I S O$, because this part of the sky was not available to this satellite.

This paper is structured as follows. First the Spitzer spectrum of NGC 2392 is presented and discussed (in Sect. 2). Then the intrinsic $\mathrm{H} \beta$ flux is determined using both the measurements of the infrared hydrogen lines and the radio continuum flux density (Sect. 3). The visible spectrum of the nebula is presented in Sect. 4 together with a new reduction of the ultraviolet (IUE) spectrum. This is followed by a discussion of the nebular electron temperature and density and the chemical composition of NGC 2392 (Sect. 4). A comparison of the resulting abundances with those in the literature is given in Sect. 5. In Sect. 6 the central star is discussed especially in relation to the nebular spectrum. In Sect. 7 a general discussion and concluding remarks are given.

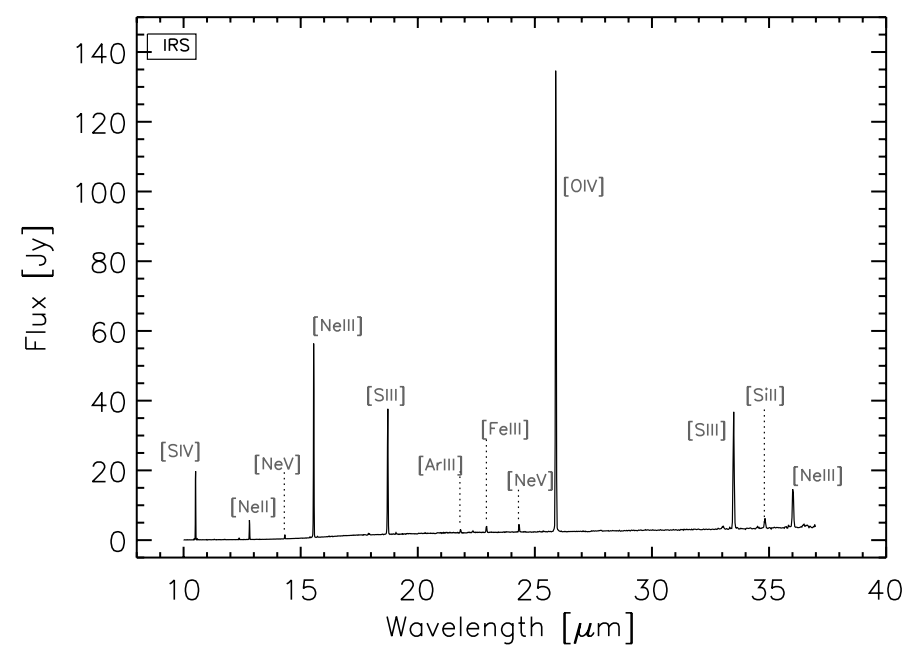

Fig. 2. Observed Spitzer-IRS high-resolution spectrum of NGC 2392. The most prominent lines are labeled in the figure.

\section{The infrared spectrum}

\subsection{IRS observations of NGC 2392}

The observations of NGC 2392 were made using the Infrared Spectrograph (IRS, Houck et al. 2004) on board the Spitzer Space Telescope with AORkeys of 4108544 (on target) and 4108800 (background). The data were processed using the s14.0 version of the pipeline and using a script version of Smart (Higdon et al. 2004). The reduction started from the droop images which are equivalent to the $b c d$ images but lack stray-cross removal and flatfield. The tool irsclean was used to remove rogue pixels. The different cycles for a given module were combined to enhance the $\mathrm{S} / \mathrm{N}$. At this point the background images were subtracted to remove the sky contribution. Then the resulting HR images were extracted using full aperture measurements. The final spectrum is shown in Fig. 2.

The great advantage of the IRS high resolution spectra compared to the ISO SWS spectra is the very high sensitivity of the IRS. Otherwise the two instruments are comparable. The IRS high resolution spectra have a spectral resolution of about 600 . The IRS high resolution instrument measures in two spectral ranges with two different modules: the short high module ( $\mathrm{SH}$ ) from $9.9 \mu \mathrm{m}$ to $19.6 \mu \mathrm{m}$ and the long high module (LH) from $18.7 \mu \mathrm{m}$ to $37.2 \mu \mathrm{m}$. The $\mathrm{SH}$ has a slit size of $4.7^{\prime \prime} \times 11.3^{\prime \prime}$, while the LH is $11.1^{\prime \prime} \times 22.3^{\prime \prime}$.

The IRS measurement of NGC 2392 was centered at $\operatorname{RA}(2000) 07^{\mathrm{h}} 29^{\mathrm{m}} 10.77^{\mathrm{s}}$ and $\operatorname{Dec}(2000)+20^{\circ} 54^{\prime} 42.5^{\prime \prime}$. This is almost the same as the value measured by Kerber et al. (2003) of RA(2000) $07^{\mathrm{h}} 29^{\mathrm{m}} 10.768^{\mathrm{s}}$ and $\operatorname{Dec}(2000)-28^{\circ} 54^{\prime} 42.44^{\prime \prime}$. Thus the IRS measurement was well centered on the nebula and both the LH and SH diaphragms measured the inner, brighter nebula. Since the LH diaphragm is larger, more of the nebula is seen in this diaphragm and therefore a correction must be made to bring the two measurements to the same scale. This was done by making use of the fact that the two spectrographs had a small wavelength region in common at about $19 \mu \mathrm{m}$. To make the continuum emission at this wavelength equal, the $\mathrm{SH}$ emission had to be increased by 4.3 . This is close to the difference in area between the two slits (4.7). The measured emission line intensities are given in Table 1, after correcting the $\mathrm{SH}$ measurements by the factor 4.3 in the column labeled "intensity". The fluxes were measured using the Gaussian line-fitting routine and derived for each nod position independently. The uncertainty in the fluxes 
Table 1. IRS spectrum of NGC 2392. The measured line intensity is given in Col. 3. The last column gives the ratio of the line intensity to $\mathrm{H} \beta(=100)$.

\begin{tabular}{|c|c|c|c|}
\hline Identification & $\lambda(\mu \mathrm{m})$ & Intensity $^{\dagger}$ & $\mathrm{I} / \mathrm{H} \beta$ \\
\hline [Ar II] & 6.989 & $18.3 \pm 3.5$ & 0.64 \\
\hline $\mathrm{HI}(6-5)$ & 7.481 & $47.5 \pm 2.2$ & 1.66 \\
\hline [Ar III] & 8.995 & $231 \pm 8.8$ & 8.05 \\
\hline [S IV] & 10.511 & $950 \pm 56$ & 33.0 \\
\hline $\mathrm{HI}(9-7)$ & 11.309 & $8.12 \pm 0.47$ & 0.28 \\
\hline [Cl IV] & 11.762 & $4.69 \pm 0.60$ & 0.163 \\
\hline H I $(7-6+11-8)$ & 12.374 & $25.3 \pm 1.4$ & \\
\hline [Ne II] & 12.815 & $220 \pm 10$ & 8.63 \\
\hline [Ar V] & 13.096 & $<2.6$ & \\
\hline$[\mathrm{Ne} \mathrm{V}]$ & 14.323 & $44.6 \pm 0.86$ & 1.56 \\
\hline [Ne III] & 15.556 & $1930 \pm 30.8$ & 67.2 \\
\hline Н I $10-8$ & 16.219 & $4.2 \pm 2.0$ & 0.146 : \\
\hline [P III] $]$ & 17.893 & $16 \pm 20$ & $0.56:$ \\
\hline$[\mathrm{Fe}$ II $]$ & 17.943 & $10.0 \pm 40$ & $0.35:$ \\
\hline [S III] & 18.718 & $1098 \pm 27$ & 38.2 \\
\hline [Cl IV] & 20.319 & $4.91 \pm 0.36$ & 0.167 \\
\hline [Ar III] & 21.827 & $25.6 \pm 1.96$ & 0.89 \\
\hline [Fe III] & 22.934 & $44.7 \pm 1.37$ & 1.55 \\
\hline$[\mathrm{Ne} \mathrm{V}]$ & 24.326 & $44.5 \pm 3.25$ & 1.55 \\
\hline [O IV] & 25.895 & $2634 \pm 119$ & 88.4 \\
\hline Н I 9-8 & 27.806 & $6.76 \pm 1.69$ & 0.236 \\
\hline$[\mathrm{Fe} \mathrm{III}]$ & 33.043 & $14.7 \pm 1.31$ & 0.511 \\
\hline [S III] & 33.487 & $530 \pm 11.5$ & 18.4 \\
\hline [Si II] & 34.825 & $45.4 \pm 1.69$ & 1.58 \\
\hline [Ne III] & 35.941 & $97.7 \pm 115:$ & 3.42: \\
\hline
\end{tabular}

$\dagger$ Intensities measured in units of $10^{-14} \mathrm{erg} \mathrm{cm}^{-2} \mathrm{~s}^{-1}$. The intensities below $19 \mu \mathrm{m}$ have been increased by a factor of 4.3 to bring them on the same scale as the LH intensities measured through a larger diaphragm. The intensities below $10 \mu \mathrm{m}$ are measured with the low resolution instrument.

(:) Indicates an uncertain value.

was assumed to be the larger of either the difference between the flux in the nod positions, or the uncertainty in the fit. The last column gives the ratio of the intensity to $\mathrm{H} \beta$ where the $\mathrm{H} \beta$ is found from the strongest hydrogen line(s) measured in the IRS spectrum. It has been assumed that the ratio of the sum of the two hydrogen lines at $12.372 \mu \mathrm{m}$ to $\mathrm{H} \beta$ has a value of $8.80 \times 10^{-3}$, which is the value given by Hummer \& Storey (1987) for an electron temperature of $15000 \mathrm{~K}$. No correction for extinction is made since it is very small. This means that the value of $\mathrm{H} \beta$ through the LH diaphragm is $2.87 \times 10^{-11} \mathrm{erg} \mathrm{cm}^{-2} \mathrm{~s}^{-1}$ and the correction for reddening has been included. Note that the three lines with wavelength less than $10 \mu \mathrm{m}$ are measured in the low resolution spectrograph.

There are other ways of determining the factor that accounts for the emission missing in the SH diaphragm. The method given above, making use of the fact that the continua in the region of wavelength overlap should be equal, is theoretically appropriate. This is because the continuum is due primarily to dust which probably has the same properties throughout the nebula. But to check this any pair of lines can be used as long as they originate from the same ion and two other conditions exist. One is that each of the lines is measured in a different diaphram. The other is that the theoretical value of the ratio of the two lines is only weakly dependent on the electron temperature and density. Five candidate pairs of lines exist. One is the ratio of the [Ne III] lines at $\lambda 15.5$ and $\lambda 36.0 \mu \mathrm{m}$. This cannot be used in this case because the $\lambda 36.0 \mu \mathrm{m}$ line is very uncertain. The [Ne V] and [Cl IV] ions both have a pair of lines, one of which is in the $\mathrm{SH}$ range and one of which is in the $\mathrm{LH}$ range. The predicted line ratio is only slightly dependent on the nebular properties so that they may be used; they are consistent with the value of 4.3. The hydrogen lines are rather weak but they also are consistent with a value of 4.3 with an error of about $20 \%$. It thus appears that the total emission in the $\mathrm{SH}$ wavelength band is obtained by increasing the measured intensities by a factor of $4.3 \pm 0.2$. This is close to the ratio of the areas of the two diaphragms, which is 4.6.

\section{Extinction}

There are several methods for obtaining the extinction: (1) comparison of radio emission with $\mathrm{H} \beta$ flux, (2) comparison of observed and theoretical Balmer decrement, (3) dip at $\lambda 2200 \AA$, (4) photometry of the exciting star. All of these methods are applicable here. First we will discuss the radio emission and how the $\mathrm{H} \beta$ flux is obtained.

\subsection{The $6 \mathrm{~cm}$ radio emission}

The $6 \mathrm{~cm}$ flux density has been measured by Becker et al. (1991) and Gregory \& Condon (1991). Becker et al. (1991) find a value of 242 mJy and Gregory \& Condon (1991) give $246 \mathrm{mJy}$. The nebula has also been measured at $21 \mathrm{~cm}$ by Condon \& Kaplan (1998) who find a value of $280.9 \mathrm{mJy}$, which implies a $6 \mathrm{~cm}$ flux density of $238 \mathrm{mJy}$ if the nebula is optically thin. We will use a value of $242 \mathrm{mJy}$. Using values of $T_{\mathrm{e}}$ and helium abundance determined below together with the equation quoted in Pottasch (1984) this implies an $\mathrm{H} \beta$ flux of $6.83 \times 10^{-11} \mathrm{erg} \mathrm{cm}^{-2} \mathrm{~s}^{-1}$, indicating that about $42 \%$ of the nebula is being measured in the LH slit.

\subsection{Extinction}

The extinction determined from the Balmer decrement differs somewhat according to different authors. Henry et al. (2000) give $C=0$, Aller \& Czyzak (1979) give $C=0.15$, Barker (1991) finds $C=0.12$.

The photometry of the star gives a higher extinction. Using $B=10.38$ and $V=10.53, B-V=-0.15$. The intrinsic value of $B-V$ for a hot star is -0.32 giving a value of $E_{B-V}=0.17$ or $C=0.25$. The extinction found from the $\lambda 2200 \AA$ dip is $E_{B-V}=$ 0.08 or $C=0.12$ (Pottasch et al. 1978).

The measured value of the integrated $\mathrm{H} \beta$ flux is $4.07 \times$ $10^{-11} \mathrm{erg} \mathrm{cm}^{-2} \mathrm{~s}^{-1}$ (Cahn et al. 1992). Using the value of $\mathrm{H} \beta$ from the radio measurements leads to a value of $C=0.225$ or $E_{B-V}=0.154$. This is slightly higher than the values found from the Balmer decrement and the $\lambda 2200 \AA$ dip but it agrees with the extinction found from the stellar photometry. We find that the radio/ $\mathrm{H} \beta$ method is the most accurate determination, so that this value will be used when necessary in this paper. But the extinction is so low that it does not affect any results in this paper.

\subsection{The visual spectrum}

The visual spectrum has been measured by several authors. The highest resolution spectrum is by Henry et al. (2000), and reliable spectra have also been reported by Barker (1991) and Aller \& Czyzak (1979). The measurements of Henry et al. (2000) and those of Barker (1991) are made at several different places in the nebula but there do not appear to be important differences between them. The results are shown in Table 2 where Cols. 3-5 give the intensities measured by the various authors relative to 
Table 2. Visual spectrum of NGC 2392.

\begin{tabular}{|c|c|c|c|c|c|}
\hline \multirow{2}{*}{$\begin{array}{c}\lambda \\
(\AA)\end{array}$} & \multirow[t]{2}{*}{ Ion } & \multicolumn{3}{|c|}{ Intensities $^{\dagger}$} & \multirow{2}{*}{$\begin{array}{l}\text { Average } \\
\text { Intens. }\end{array}$} \\
\hline & & (1) & (2) & (3) & \\
\hline $3727^{*}$ & {$[\mathrm{O} \mathrm{II}]$} & 107 & 120 & 108 & 110 \\
\hline 3869 & {$[\mathrm{Ne}$ III] } & 100 & 130 & 90 & 105 \\
\hline 4101 & $\mathrm{H} \delta$ & 24.0 & 26.5 & 25.2 & 25.2 \\
\hline 4267 & C II & & 0.3: & & 0.3 : \\
\hline 4340 & $\mathrm{H} \gamma$ & 46.0 & 48.0 & 48.0 & 47.2 \\
\hline 4363 & [O III] & 14 & 25 & 19.5 & 19.5 \\
\hline 4686 & He II & 38 & 38 & 35 & 37 \\
\hline 4740 & [Ar IV] & & 2.2 & 2.5 & 2.3 \\
\hline 4861 & $\mathrm{H} \beta$ & 100 & 100 & 100 & 100 \\
\hline 5007 & {$[\mathrm{O} \mathrm{III}]$} & 950 & 1200 & 1260 & 1150 \\
\hline 5517 & [Cl III] & 0.6 & 0.72 & 0.65 & 0.65 \\
\hline 5538 & [Cl III] & & 0.68 & 0.50 & 0.59 \\
\hline 5755 & {$[\mathrm{~N}$ II $]$} & 2.0 & 1.6 & 1.51 & 1.6 \\
\hline 5876 & $\mathrm{He} \mathrm{I}$ & 6.8 & 7.8 & 7.45 & 7.4 \\
\hline 6312 & [S III] & 2.5 & 3.6 & 3.3 & 3.2 \\
\hline 6563 & $\mathrm{H} \alpha$ & 275 & 295 & 283 & 285 \\
\hline 6584 & {$[\mathrm{~N}$ II $]$} & 95 & 95 & 85.5 & 92 \\
\hline 6717 & {$[\mathrm{~S}$ II $]$} & 8.0 & 6.7 & 4.8 & 6.7 \\
\hline 6731 & {$[\mathrm{~S} \mathrm{II}]$} & 10.0 & 7.9 & 7.8 & 8.6 \\
\hline 7005 & {$[\mathrm{ArV}]$} & & & 0.15 & 0.15 \\
\hline 7135 & [Ar III] & 14 & 14.3 & 12.3 & 14 \\
\hline 7237 & {$[\mathrm{Ar} I \mathrm{IV}]$} & & & 0.19: & 0.19: \\
\hline 8045 & {$[\mathrm{Cl}$ IV $]$} & 0.4 & & 0.47 & 0.45 \\
\hline 9532 & [S III] & 91 & & & 91 \\
\hline
\end{tabular}

$\dagger$ References; (1) Henry et al. (2000), (2) Barker (1991), (3) Aller \& Czyzak (1979).

(:) Indicates uncertain values.

* This is a blend of $\lambda 3726$ and $\lambda 3729$ lines.

$\mathrm{H} \beta=100$ for the lines of interest. The line intensities have been corrected by the individual authors for extinction. Henry et al. (2000) felt that no extinction correction was necessary, the other authors using the reddening curve of Seaton (1979) find the extinctions listed in the previous section. No attempt has been made to use a common extinction correction because all give a correct Balmer decrement. In the last column average values are given; more weight is given to the measurements of the first two authors. All authors estimate that the strongest lines have a $10 \%$ error, the intermediate strength lines (about $5 \%$ of $\mathrm{H} \beta$ ) have about $20 \%$ error and the weakest lines have about $30 \%$ error. The average intensities have about the same error.

\subsection{The IUE ultraviolet spectrum}

There are 80 low resolution IUE observations of this nebula as well as six high resolution observations. Most were taken with the large aperature $\left(10^{\prime \prime} \times 23^{\prime \prime}\right)$ with varying exposure times, but quite a few were taken with a small aperature ( $3^{\prime \prime}$ diameter). The small aperature measurements were used by Barker (1991), who did not find any important abundance variations in the nebula. We find the small aperature measurements are too noisy and we do not use them. The large aperture measurements do not cover the entire nebula. Most of the measurements which exclude the central star cover about $10 \%$ to $15 \%$ of the nebula. We have used three of the highest $\mathrm{S} / \mathrm{N}$ observations made with long exposure times. These are SWP05231 and SWP25236 for the short wavelength region and LWR04515 for the long wavelength region. We have checked that the strongest lines are not saturated by measuring these same lines on spectra with shorter exposure times. They are shown in Table 3. The uncertainties are
Table 3. IUE Spectrum of NGC 2392.

\begin{tabular}{clccc}
\hline \hline$\lambda$ & Ion & \multicolumn{3}{c}{ Intensities } \\
\cline { 3 - 5 }$(\AA)$ & & $(1)$ & $(2)$ & $(\mathrm{I} / \mathrm{H} \beta)$ \\
\hline 1335 & C II & 10.2 & 11.0 & 16.0 \\
1400 & O IV & 36.3 & 36.6 & 53.4 \\
1485 & N IV] & 24.8 & 24.2 & 35.2 \\
1548 & C IV & 77.7 & 75.5 & 110 \\
1640 & He II & 185 & 176 & 256 \\
1663 & O III] & 53.8 & 34.6 & 50.4 \\
1750 & N III] & 49.4 & 45.4 & 66.1 \\
1881 & Si III] & 14.7 & 14.0 & 20.4 \\
1890 & Si III] & 12.0 & 11.4 & 16.6 \\
1909 & C III] & 156 & 148 & 216 \\
2325 & C II] & 36.4 & 31.8 & 46.4 \\
2423 & [Ne IV] & 187 & 145 & 211 \\
2472 & {$[\mathrm{O} \mathrm{III]}$} & 20 & 14.7 & 21.5 \\
2512 & He II & 24.2 & 17.3 & 25.2 \\
\hline
\end{tabular}

(1) Measured intensity from low resolution spectra SWP05231 and LWR04515 in units of $10^{-13} \mathrm{erg} \mathrm{cm}^{-2} \mathrm{~s}^{-1}$.

(2) Intensity corrected for diaphragm size and extinction in units of $10^{-12} \mathrm{erg} \mathrm{cm}^{-2} \mathrm{~s}^{-1}$. I/H $\beta$ is normalized to $\mathrm{H} \beta=100$.

about the same as given by Henry et al. (2000): about $10 \%$ for the strongest lines and about $25 \%$ for the weaker lines.

The extinction correction was made by assuming a theoretical ratio for the He II line ratio $\lambda 1640 / \lambda 4686 \AA$ at $T=12500 \mathrm{~K}$ and an $N_{\mathrm{e}}$ of $10^{3} \mathrm{~cm}^{-3}$. The ratio of $\lambda 1640$ to $\mathrm{H} \beta$ can then be found using the $\lambda 4686 / \mathrm{H} \beta$ ratio in Table 2 which leads to a value of the $\lambda 1640 \AA$ line as given in Col. (2) of Table 3. A further correction for extinction relative to $\lambda 1640 \AA$ is then made using the reddening curve of Fluks et al. (1994) but because of the small extinction this is never more than $20 \%$, which indicates the uncertainties of the UV intensities above the errors of measurement given above. The results are shown in the last two columns of Table 3.

\section{Chemical composition of the nebulae}

The method of analysis is the same as used in the papers cited in the introduction. First the electron density and temperature as a function of the ionization potential are determined. Then the ionic abundances are determined, using density and temperature appropriate for the ion under consideration. Then the element abundances are found for those elements in which a sufficient number of ionic abundances have been derived.

\subsection{Electron density}

The ions used to determine $N_{\mathrm{e}}$ are listed in the first column of Table 4. The ionization potential required to reach this stage of ionization, and the wavelengths of the lines used, are given in Cols. 2 and 3 of the table. Note that the wavelength units are $\AA$ when 4 figures are given and microns when 3 are shown. The observed ratio of the lines is given in the fourth column; the corresponding $N_{\mathrm{e}}$ is given in the fifth column. The temperature used is discussed in the following section, but is unimportant since these line ratios are essentially determined by the density. No density is given from the Ne III lines because of the uncertainty of the $36 \mu \mathrm{m}$ line intensity. The density from the C III] lines is uncertain because it is possible that the stronger of the two lines may be saturated. 
Table 4. Electron density indicators in NGC 2392.

\begin{tabular}{lcccc}
\hline \hline Ion & $\begin{array}{c}\text { Ioniz. } \\
\text { Pot.(eV) }\end{array}$ & $\begin{array}{c}\text { Lines } \\
\text { Used }\end{array}$ & $\begin{array}{c}\text { Observed } \\
\text { Ratio }\end{array}$ & $\begin{array}{c}N_{\mathrm{e}} \\
\left(\mathrm{cm}^{-3}\right)\end{array}$ \\
\hline$[\mathrm{S} \mathrm{II}]$ & 10.4 & $6731 / 6716$ & 1.28 & 1600 \\
{$[\mathrm{O} \mathrm{II}]$} & 13.6 & $3626 / 3729$ & 1.35 & 1650 \\
{$[\mathrm{~S} \mathrm{III}]$} & 23.3 & $33.5 / 18.7$ & 0.48 & 2600 \\
{$[\mathrm{Cl}$ III $]$} & 23.8 & $5538 / 5518$ & 0.86 & 1200 \\
$\mathrm{C} \mathrm{III}]$ & 24.4 & $1906 / 1909$ & 1.46 & $1000:$ \\
{$[\mathrm{Ne} \mathrm{IV}]$} & 63.5 & $2425 / 2422$ & 1.4 & $1000:$ \\
{$[\mathrm{Ne} \mathrm{V}]$} & 97.1 & $24.3 / 14.3$ & 0.99 & $1000:$ \\
\hline
\end{tabular}

(:) Indicates uncertain values.

There is no indication that the electron density varies with ionization potential in a systematic way. The electron density appears to be about $1200 \mathrm{~cm}^{-3}$. The error is about $20 \%$. It is interesting to compare this value of the density with the rms density found from the $\mathrm{H} \beta$ line. This depends on the distance of the nebula which is not accurately known, and on the angular size of the nebula. For this calculation we use a distance of $1.5 \mathrm{kpc}$ and a diameter of $18^{\prime \prime}$ for the inner region. This gives an rms density of $2000 \mathrm{~cm}^{-3}$ for the inner region. The comparison is difficult because the measurement may include some $\mathrm{H} \beta$ flux from the outer region which will lower the density. This uncertainty is illustrated by two different O II measurements. Kingsburgh \& Barlow (1994) measured a $3726 / 3729 \AA=1.35$ at the center of the nebula, corresponding to a density of $1650 \mathrm{~cm}^{-3}$, while O'Dell \& Castaneda (1984) measuring at the (brighter) rim of the nebula find a value of 1.78 for the ratio, corresponding to a density of $3000 \mathrm{~cm}^{-3}$. We will use a density of $1500 \mathrm{~cm}^{-3}$ in further discussion of the abundances, but any value between $1000 \mathrm{~cm}^{-3}$ and $3000 \mathrm{~cm}^{-3}$ will give the same values of abundance.

\subsection{Electron temperature}

A number of ions have lines originating from energy levels far enough apart that their ratio is sensitive to the electron temperature. These are listed in Table 5 which is arranged similarly to the previous table. The values are also shown in Fig. 3 where it can be seen that the electron temperature has a strong gradient as a function of ionization potential. Other PNe have also shown a gradient of electron temperature but it is especially strong in this nebula, going from a value of about $10000 \mathrm{~K}$ for the lowest ionization potentials (presumably formed in the outer regions of the nebula) through a value of 14000 to $15000 \mathrm{~K}$ at an ionization potential of about $35 \mathrm{eV}$, to a value well over $20000 \mathrm{~K}$ at ionization potentials above $50 \mathrm{eV}$. For these latter ions it is impossible to specify the temperature accurately, so that the abundance of these ions can only be determined from the infrared lines since the population of the lower levels is only slightly dependent on the temperature.

\subsection{Ion and element abundances}

The ion abundances have been determined using the following equation:

$\frac{N_{\text {ion }}}{N_{\mathrm{p}}}=\frac{I_{\text {ion }}}{I_{\mathrm{H}_{\beta}}} N_{\mathrm{e}} \frac{\lambda_{\mathrm{ul}}}{\lambda_{\mathrm{H}_{\beta}}} \frac{\alpha_{\mathrm{H}_{\beta}}}{A_{\mathrm{ul}}}\left(\frac{N_{\mathrm{u}}}{N_{\text {ion }}}\right)^{-1}$

where $I_{\text {ion }} / I_{\mathrm{H}_{\beta}}$ is the measured intensity of the ionic line compared to $\mathrm{H} \beta, N_{\mathrm{p}}$ is the density of ionized hydrogen, $\lambda_{\mathrm{ul}}$ is the wavelength of the line, $\lambda_{\mathrm{H}_{\beta}}$ is the wavelength of $\mathrm{H} \beta, \alpha_{\mathrm{H}_{\beta}}$ is the
Table 5. Electron temperature indicators in NGC 2393.

\begin{tabular}{lcccc}
\hline \hline Ion & $\begin{array}{c}\text { Ioniz. } \\
\text { Pot.(eV) }\end{array}$ & $\begin{array}{c}\text { Lines } \\
\text { Used }\end{array}$ & $\begin{array}{c}\text { Observed } \\
\text { Ratio }\end{array}$ & $\begin{array}{c}T_{\mathrm{e}} \\
(\mathrm{K})\end{array}$ \\
\hline$[\mathrm{N} \mathrm{II}]$ & 14.5 & $5755 / 6584$ & 0.017 & 10000 \\
{$[\mathrm{~S} \mathrm{III}]$} & 23.3 & $6312 / 18.7$ & 0.0 .084 & 12000 \\
{$[\mathrm{Ar}$ III $]$} & 27.6 & $7136 / 21.8$ & 15.8 & 10000 \\
{$[\mathrm{O} \mathrm{III}]$} & 35.1 & $4363 / 5007$ & 0.0165 & 14700 \\
{$[\mathrm{O}$ III $]$} & 35.1 & $1663 / 5007$ & 0.0496 & 15200 \\
{$[\mathrm{Ne}$ III $]$} & 41.0 & $3869 / 15.5$ & 1.56 & 13500 \\
{$[\mathrm{O} \mathrm{IV}]$} & 54.9 & $1400 / 25.9$ & 0.44 & $22000:$ \\
{$[\mathrm{Ne}$ $]$} & 97.1 & $3425 / 24.3$ & $2.2:$ & 25000 \\
\hline
\end{tabular}

(:) Indicates uncertain value.

When the wavelength has 4 figures it has the units of Angstrom and 3 figures is micron.

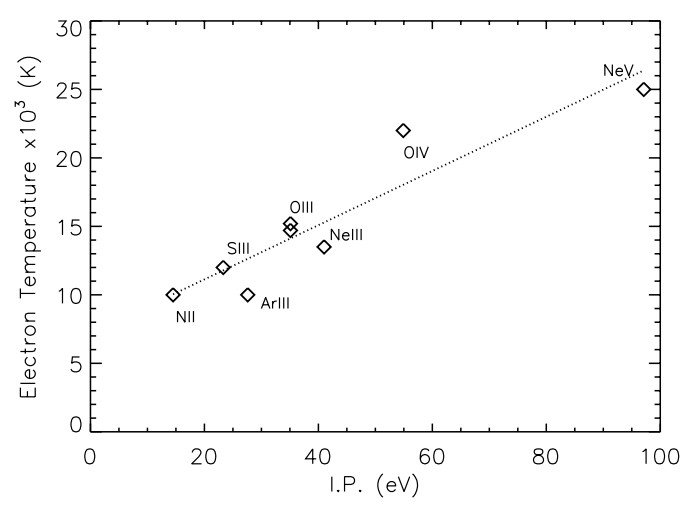

Fig. 3. Electron temperature is plotted as a function of the ionization potential.

effective recombination coefficient for $\mathrm{H} \beta, A_{\mathrm{ul}}$ is the Einstein spontaneous transition rate for the line, and $N_{\mathrm{u}} / N_{\text {ion }}$ is the ratio of the population of the level from which the line originates to the total population of the ion. This ratio has been determined using a five level atom. The atomic data used is given in the paper of Pottasch \& Beintema (1999). The use of a 5 level atom in the case of iron is justified because collisional rates to higher levels followed by cascade through the lower levels are quite small in comparison to direct collisions to the lower levels.

The results are given in Table 6, where the first column lists the ion concerned, the second column the line used for the abundance determination, and the third column gives the intensity of the line used relative to $\mathrm{H} \beta=100$. The fourth column gives the value of the electron temperature used for the abundance determination of the ion, which is shown in the fifth column. This temperature is determined by plotting the temperatures given in Table 5 as a function of ionization potential, drawing a line through these points as shown in Fig. 3, and then reading the temperature of the ion under consideration from its ionization potential. The fifth column gives the ionic abundance assuming the ion is formed at this temperature, while the sixth column gives the ionization correction factor (ICF), which has been determined empirically. Notice that the ICF is unity (or almost unity) for all elements listed in the table except for $\mathrm{Fe}, \mathrm{Si}, \mathrm{Cl}$ and $\mathrm{P}$.

The error of measurement of the IRS intensities as can be seen in Table 1 is usually small, often not more than $5-7 \%$. In the few cases when the error is large this has either been indicated with a ":" or by not using the line. The correction for adjusting the $\mathrm{SH}$ to $\mathrm{LH}$ intensity scales and the diaphragm size is probably small, about $10 \%$. This includes the assumption that 
Table 6. Ionic concentrations and chemical abundances in NGC 2392. Wavelengths in Angstrom for all values of $\lambda$ above 1000, otherwise in $\mu \mathrm{m}$.

\begin{tabular}{|c|c|c|c|c|c|c|}
\hline Ion & $\lambda$ & $\mathrm{I} / \mathrm{H} \beta$ & $T_{\mathrm{e}}$ & $N_{\text {ion }} / N_{\mathrm{p}}$ & ICF & $N_{\text {el. }} / N_{\mathrm{p}}$ \\
\hline $\mathrm{He}^{+}$ & 5875 & 7.4 & 12000 & 0.048 & & \\
\hline $\mathrm{He}^{++}$ & 4686 & 37 & 14000 & 0.032 & 1.0 & 0.080 \\
\hline $\mathrm{C}^{+}$ & 2324 & 41.7 & 10000 & $7.5(-5)$ & & \\
\hline $\mathrm{C}^{++}$ & 1909 & 240 & 11500 & $2.3(-4)$ & & \\
\hline $\mathrm{C}^{+3}$ & 1548 & 115 & 17000 & $1.7(-5)$ & 1.0 & $3.3(-4)$ \\
\hline $\mathrm{N}^{+}$ & 6584 & 92 & 10000 & $1.8(-5)$ & & \\
\hline $\mathrm{N}^{++}$ & 1750 & 62.3 & 12500 & $1.5(-4)$ & & \\
\hline $\mathrm{N}^{+3}$ & 1485 & 30 & 17000 & $1.4(-5)$ & 1.0 & $1.85(-4)$ \\
\hline $\mathrm{O}^{+}$ & 3727 & 110 & 10000 & $5.0(-5)$ & & \\
\hline $\mathrm{O}^{++}$ & 5007 & 1150 & 14000 & $1.9(-4)$ & & \\
\hline $\mathrm{O}^{++}$ & 1663 & 57 & 14000 & $2.3(-4)$ & & \\
\hline $\mathrm{O}^{+3}$ & 25.8 & 88.4 & 19500 & $2.3(-5)$ & 1.0 & $2.9(-4)$ \\
\hline $\mathrm{Ne}^{+}$ & 12.8 & 8.63 & 11000 & $1.2(-5)$ & & \\
\hline $\mathrm{Ne}^{++}$ & 15.5 & 67.2 & 15000 & $4.7(-5)$ & & \\
\hline $\mathrm{Ne}^{++}$ & 3869 & 105 & 15000 & $3.5(-5)$ & & \\
\hline $\mathrm{Ne}^{+3}$ & 2423 & 211 & 21000 & $2.4(-5)$ & & \\
\hline $\mathrm{Ne}^{+4}$ & 24.3 & 1.55 & 27000 & $1.9(-7)$ & 1.0 & $8.5(-4)$ \\
\hline $\mathrm{S}^{+}$ & 6731 & 8.6 & 9000 & $4.9(-7)$ & & \\
\hline $\mathrm{S}^{++}$ & 18.7 & 38.2 & 11300 & $3.5(-6)$ & & \\
\hline $\mathrm{S}^{++}$ & 6312 & 3.2 & 11300 & $5.2(-6)$ & & \\
\hline $\mathrm{S}^{+3}$ & 10.5 & 33.0 & 14000 & $0.87(-6)$ & 1.0 & $5.0(-6)$ \\
\hline $\mathrm{Ar}^{++}$ & 21.8 & 0.89 & 12000 & $1.3(-6)$ & & \\
\hline $\mathrm{Ar}^{++}$ & 7135 & 14.0 & 12000 & $1.0(-6)$ & & \\
\hline $\mathrm{Ar}^{+3}$ & 4740 & 2.3 & 15300 & $2.9(-7)$ & & \\
\hline $\mathrm{Ar}^{+3}$ & 7237 & 0.19 & 15300 & 8.1(-7): & & \\
\hline $\mathrm{Ar}^{+4}$ & 7005 & 0.15 & 20500 & $1.4(-8)$ & 1.2 & $2.2(-6)$ \\
\hline $\mathrm{Cl}^{++}$ & 5538 & 0.59 & 11400 & $7.2(-8)$ & & \\
\hline $\mathrm{Cl}^{+3}$ & 20.3 & 0.167 & 14800 & $0.88(-8)$ & & \\
\hline $\mathrm{Cl}^{+3}$ & 8045 & 0.45 & 14800 & $2.7(-8)$ & 1.3 & $1.3(-7)$ \\
\hline $\mathrm{Fe}^{++}$ & 22.9 & 1.55 & 10500 & $4.7(-7)$ & & \\
\hline $\mathrm{Fe}^{++}$ & 33.0 & 0.51 & 10500 & $5.8(-7)$ & 1.6 & $8.0(-7)$ \\
\hline $\mathrm{Si}^{+}$ & 34.8 & 1.58 & 9000 & $3.8(-7)$ & & \\
\hline $\mathrm{Si}^{++}$ & 1888 & 37.0 & 10400 & $1.4(-5)$ & 1.3 & $1.9(-5)$ \\
\hline $\mathrm{P}^{++}$ & 17.9 & 0.56 & 10500 & $3.4(-8)$ & 1.9: & $6.5(-8)$ : \\
\hline $\mathrm{C}^{++}$ & 4267 & 0.3 & 11500 & $3.4(-4)$ & & \\
\hline
\end{tabular}

Intensities given with respect to $\mathrm{H} \beta=100$.

(:) Indicates uncertain value.

the unmeasured parts of the nebula have the same spectrum as the measured parts. This has been checked in the optical region by Barker (1991) and can be seen to be a reasonable approximation in the ultraviolet region by comparing the IUE spectra obtained in different parts of the nebula. The uncertainty of the collisional strengths introduces an error of $10-15 \%$ so that the total error for the ions of neon, sulfur and argon determined with the IRS measurements is less than $20 \%$. This will also be true of the abundances of these elements because the ICF for these elements is close to unity. The error for the nitrogen and oxygen abundances is somewhat higher because the visual and ultraviolet measurements are less certain. In addition the temperature is more important for these ions and the total errors may be twice as large. The element abundances are given in the last column, The carbon recombination line abundance is given at the end of the table. These abundances will be discussed in the next sections.

The helium abundance was derived using the theoretical work of Benjamin et al. (1999). For recombination of singly ionized helium, most weight is given to the $\lambda 5875 \AA$ line, because the theoretical determination of this line is the most reliable.
Table 7. Comparison of abundances in NGC 2392.

\begin{tabular}{lrrrr}
\hline \hline Elem. & Present & $\mathrm{H}^{\dagger}$ & $\mathrm{B}^{\dagger}$ & Solar $^{\dagger}$ \\
\hline $\mathrm{He}$ & 0.080 & 0.076 & 0.097 & 0.098 \\
$\mathrm{C}(-4)$ & 3.3 & 2.2 & 0.42 & 2.5 \\
$\mathrm{~N}(-4)$ & 1.85 & 1.1 & 1.1 & 0.60 \\
$\mathrm{O}(-4)$ & 2.9 & 2.8 & 3.4 & 4.6 \\
$\mathrm{Ne}(-5)$ & 8.5 & 6.4 & 7.6 & 12 \\
$\mathrm{~S}(-6)$ & 5.0 & & 4.3 & 14 \\
$\mathrm{Ar}(-6)$ & 2.2 & & 1.4 & 4.2 \\
$\mathrm{Cl}(-7)$ & 1.3 & & & 3.5 \\
$\mathrm{Si}(-6)$ & 19 & & & 32 \\
$\mathrm{Fe}(-7)$ & 8.0 & & & 280 \\
$\mathrm{P}(-8)$ & 6.5 & & & 23 \\
\hline
\end{tabular}

${ }^{\dagger}$ References: H: Henry et al. (2000), B: Barker (1991), Solar: Asplund et al. (2005), except Ne and Ar (see Pottasch \& Bernard-Salas 2006).

\section{Comparison with other abundance determinations}

Table 7 shows a comparison of our abundances with the most important determinations in the past 20 years. Only about half of the elements have been reported before. Good agreement is found for oxygen; this is because the same electron temperature is used for the most important oxygen ion. For the other elements the agreement is less good. We find a rather higher carbon abundance, much higher than found by Barker (1991) and slightly higher than found by Henry et al. (2000). A C/O ratio greater than unity is found but the error is large enough that it could be slightly less than unity. Nitrogen is somewhat higher than found by the other authors and clearly a factor of two higher than the solar abundance. Most of the other elements are consistent with a galactocentric abundance gradient $(-0.085 \mathrm{dex} / \mathrm{kpc})$ as described for example by Pottasch \& Bernard-Salas (2006). Iron is depleted by more than a factor of 30 with respect to the solar abundance, as it is in most of the nebulae already studied. Phosphorus seems rather strongly depleted as well, but it is uncertain because of the large measurement error. The comparison made in the table with the solar abundance is taken from Asplund et al. (2005). Note that for sulfur and chlorine more weight has been given to the abundance determination in meteorites since this determination is more accurate than for the Sun itself. Neon and argon abundances are taken from the references given in Pottasch \& Bernard-Salas (2006).

The main differences with earlier work lie in the interpretation of the spectrum. First because the gradient of electron temperature has been included here. Considering that a very large temperature gradient is present the abundances are not much affected by it. This is probably because the important ions contributing to the total abundances are formed at temperatures not so far from the temperature at which the $\mathrm{O}^{++}$ion is formed. An exception to this is the $\mathrm{C}^{++}$which is formed at a lower temperature, which explains why Barker (1991) obtained a much smaller carbon abundance.

A further cause of error is the ICF, can give important errors for $\mathrm{Si}, \mathrm{Fe}$ and especially $\mathrm{P}$ for which only one ion has been observed. For these elements the ICF has been determined by comparison with the results of model calculations made for other PNe which have similar excitation. This makes the results for these elements uncertain, but since the ions observed are important contributers to the total abundance, the error even for these elements is probably less than a factor of two. 
The abundances found agree with those expected from the gradient of PNe abundances found by Pottasch \& Bernard-Salas (2006).

\subsection{Recombination line abundances of carbon}

The $\mathrm{C}^{++}$population can be obtained from the recombination line $\lambda 4267 \AA$ as well as from the collisionally excited line at $\lambda 1909 \AA$. The advantage of the recombination line is that it is not sensitive to the electron temperature, and is in the visual spectrum as well. It has the disadvantage that it is quite faint and thus difficult to measure accurately. It has been used for at least 30 years, and has been found to sometimes give higher $\mathrm{C}^{++}$populations than the collisional line. The reason for this is not clear (e.g. see the discussion of Liu et al. 2000). We have redetermined the $\mathrm{C}^{++}$abundance using using the recombination rates for this line and the result is shown in the last line of Table 6. This is essentially the same value as that determined from the $\lambda 1909 \AA$ collisional line, since the error of measurement of the weak $\lambda 4267 \AA$ line is quite large. It is therefore true that for carbon both determinations give a similar result. This is in contrast to $\mathrm{M} 1-42$ where the $\mathrm{C}^{++}$found from the recombination line is an order of magnitude higher than that found from the collisional line (Pottasch et al. 2007). Since both nebulae have almost the same electron density it may be concluded that the electron density does not contribute to this effect.

\section{Discussion of stellar evolution}

The rather low abundances of helium, oxygen, neon and sulfur are consistent with the position of the nebula in the galaxy, which is about $10 \mathrm{kpc}$ from the galactic center, assuming that the sun is $8 \mathrm{kpc}$ from the galactic center. These elements fit very well on the abundances as a function of position in the galaxy as given by Pottasch \& Bernard-Salas (2006). Especially the low helium abundance indicates that no helium has been produced which implies that the second dredge-up and hot-bottom burning have not taken place. The carbon abundance is somewhat higher than oxygen suggesting that the third dredge-up has begun. Following the models of Karakas (2003) this will occur at a stellar mass of about $1.7 M_{\odot}$. This model has increased its nitrogen abundance by about a factor of five in the first dredge-up so that it is reasonable that the actual model is to be sought in this direction.

\section{The central star}

\subsection{Stellar temperature}

As discussed in the introduction, the spectrum of the central star has been studied by several authors. Pauldrach et al. (2004) have fitted model atmospheres to the measured ultraviolet stellar spectrum and Kudritzki et al. (1997) have studied the optical spectrum. Pauldrach et al. (2004) determined the effective stellar temperature from the FeIV/FeV ionization balance to be $40000 \mathrm{~K}$ while Kudritzki et al. (1997) using the ionization equilibrium of HeI and HeII obtained a similar value of $45000 \mathrm{~K}$.

The stellar temperature can also be determined from the nebular spectrum. Enough information is available to compute both the Zanstra temperature and the Energy Balance temperature of the central star. The Zanstra temperature requires the knowledge of the stellar apparent magnitude, the extinction and the $\mathrm{H} \beta$ flux. The last two quantities have been given in Sect. 3. The apparent magnitude is listed by Acker et al. (1992) as $V=10.53$.
Assuming that the star radiates as a blackbody the hydrogen Zanstra temperature $T_{\mathrm{z}}(\mathrm{H})=37000 \mathrm{~K}$ and the ionized helium Zanstra temperature is $78000 \mathrm{~K}$. The energy balance temperature requires the knowledge of the ratio of the forbidden-line intensities to $\mathrm{H} \beta$. This value is found by summing the intensities given above, and is about 28 to 30 after making a correction for unmeasured lines using the table of Preite-Martinez \& Pottasch (1983). This could be slightly higher but not lower. To convert this value to a stellar temperature, the formulation of Preite-Martinez \& Pottasch (1983) is used, assuming blackbody radiation from the central star. The value of Case II (the nebula is optically thin to radiation which will ionize hydrogen but optically thick to ionized helium radiation) for the energy balance temperature $\left(T_{\mathrm{EB}}\right)$ is $80000 \mathrm{~K}$. If a model atmosphere had been used instead of a blackbody, the energy balance temperature could be lower. The low value of the hydrogen Zanstra temperature $\left(T_{\mathrm{Z}}(\mathrm{H})\right)$ may be due to the nebula being optically thin to radiation ionizing hydrogen. An average stellar temperature of about 75000 to $80000 \mathrm{~K}$ is a reasonable first approximation. This is also the conclusion of Tinkler \& Lamers (2002) who estimate a central star temperature of $74000 \mathrm{~K}$.

This strange behavior of the central star has been known for many years. Heap (1977) estimated the stellar spectrum as spectral class O6 giving the star a much lower temperature than the HeII Zanstra temperature. This was confirmed by Pottasch et al. (1978) who pointed out that the stellar continuum between $1500 \AA$ and $5500 \AA$ has a spectral distribution more consistent with a blackbody at $40000 \mathrm{~K}$ than one at $70000 \mathrm{~K}$. But the measured nebular spectrum showing ions of high ionization potential such as $\mathrm{O}$ IV and $\mathrm{Ne} \mathrm{V}$ indicates that there is much more far ultraviolet radiation than is consistent with a blackbody at $40000 \mathrm{~K}$, a conclusion already reached by Natta et al. (1980).

Heap (1977) suggested that there is a second star of a higher temperature which is the source of the nebular ionization. Ciardullo et al. (1999) using the HST wide field camera found a faint companion at a separation of $2.65^{\prime \prime}$ in the $I$ band but it was invisible in the $V$ band. Its properties are as yet unknown. It is also unknown if it is physically associated with the nebula. It is therefore possible that the bright star at the center of NGC 2392 is not the actual exciting star of the nebula. A determination of the radius and luminosity of such a star may be derived from Pottasch \& Acker (1989). The luminosity of a blackbody of temperature $T=80000 \mathrm{~K}$ embedded in a nebula is 105 times the luminosity of the $\mathrm{H} \beta$ flux emitted by the nebula. The luminosity of the $\mathrm{H} \beta$ flux emitted from NGC 2392, if placed at a distance of $1500 \mathrm{pc}$, is $4.7 L_{\odot}$. This leads to a total luminosity of the star $L=493 L_{\odot}$. The star must then have a radius of $R=0.116 R_{\odot}$. Such a star would have an unreddened magnitude $m(V)=14.5$. The star found by Ciardullo et al. (1999) was fainter than $m(V)=17.3$ so that this could not be the exciting star. If such an exciting star exists it must be much closer to the bright star which would make it impossible to observe with the current facilities.

\section{Conclusions}

The nebular abundances of eleven elements have been determined. It has been shown that those elements, which have not been created in stellar evolution $(\mathrm{O}, \mathrm{Ne}, \mathrm{S}, \mathrm{Ar}, \mathrm{Cl}$ and possibly $\mathrm{Si}$ ), have abundances of about a factor of two lower than the solar abundance. This is consistent with the expectation of a gradient in the stellar abundances as a function of distance from the galactic center. The helium abundance is lower than solar; this indicates that no helium has been formed during the evolution 
of the central star. Carbon has apparently been formed in a third dredge-up as it is more abundant than oxygen. Nitrogen has also been formed, probably in the first dredge-up. The abundances are consistent with the central star having evolved from an object having about $1.7 M_{\odot}$. The low iron abundance is also found in almost all PNe studied and seems to be caused by its being sequestred in dust.

The electron temperature is found to vary strongly with the ionization potential of the ion used for its determination. This indicates a strong gradient in the nebula, higher than in any PNe yet studied. The reason for this high gradient is not clear. It could be related to the fact that diffuse X-ray emission has been detected within its inner shell (Guerrero et al. 2005), which in turn may be caused by the existence of the exceptionally large expansion velocity of the inner shell.

Finally, the presence of high stages of ionization is confirmed. Both O IV and Ne V are clearly present. This seems to indicate that the stellar temperature is considerably higher that the value of $40000 \mathrm{~K}$ found from a study of the central star. The suggestion that an additional star is present is discussed, but no solution to this problem can be given.

Acknowledgements. This work is based on observations made with the Spitzer Space Telescope, which is operated by the Jet Propulsion Laboratory, California Institute of Technology under NASA contract 1407. Support for this work was provided by NASA through Contract Number 1257184 issued by JPL/Caltech.

\section{References}

Acker, A., Marcout, J., Ochsenbein, F., et al. 1992, Strasbourg-ESO catalogue Aller, L. H., \& Czyzak, S. J. 1979, Ap\&SS, 62, 397

Asplund, M., Grevesse, N., \& Sauval, A. J. 2005, ASP Conf. Ser., ed. Bash, \& Barnes
Barker, T. 1991, ApJ, 371, 217

Becker, R. H., White, R. L., \& Edwards, A. L. 1991, ApJS, 75, 1 Benjamin, R. A., Skillman, E. D., \& Smits, D. P. 1999, ApJ, 514, 307 Bernard Salas, J., Pottasch, S. R., Beintema, D. A., \& Wesselius, P. R. 2001, A\&A, 367, 949

Cahn, J. H., Kaler, J. B., \& Stanghellini, L. 1992, A\&AS, 94, 399 Ciardullo, R., Bond, H. E., Sipior, M. S., et al. 1999, AJ, 118, 488 Condon, J. J., \& Kaplan, D. L. 1998, ApJS, 117, 361

Davey, A. R., Storey, P. J., \& Kisielius, R. 2000, A\&AS, 142, 85

Fluks, M. A., Plez, B., de Winter, D., et al. 1994, A\&AS, 105, 311 Gregory, P. C., \& Condon, J. J. 1991, ApJS, 75, 1011

Guerrero, M. A., Chu, Y.-H., Gruendl, R. A., et al. 2005, A\&A, 430, L69 Heap, S. R. 1977, ApJ, 215, 864

Henry, R. B. C., Kwitter, K. B., \& Bates, J. A. 2000, ApJ, 531, 928

Higdon, S. J. U., Devost, D., Higdon, J. L., et al. 2004, PASP, 116, 975

Houck, J. R., Appelton, P. N., Armus, L., et al. 2004, ApJS, 154, 18

Hummer, D. G., \& Storey, P. J. 1987, MNRAS, 224, 801

Karakas, A. I. 2003, Thesis, Monash Univ. Melbourne

Kerber, F., Mignani, R. P., Guglielmetti, F., et al. 2003, A\&A, 408, 1029

Kingsburgh, R. L., \& Barlow, M. J. 1994, MNRAS, 271, 257

Kudritzki, R.-P., Mendez, R. H., Puls, J., \& McCarthy, J. K. 1997, IAU Symp., 180,64

Liu, X.-W., Storey, P. J., Barlow, M. J., et al. 2000, MNRAS, 312, 583

Natta, A., Pottasch, S. R., \& Preite-Martinez, A. 1980, A\&A, 84, 284

O’Dell, C. R., \& Ball, M. E. 1985, ApJ, 289, 526

O'Dell, C. R., \& Castaneda, H. O. 1984, ApJ, 283, 158

Pauldrach, A. W. A., Hoffmann, T. L., \& Mendez, R. H. 2004, A\&A, 419, 111

Pottasch, S. R. 1984, Planetary Nebulae, Reidel Publ. Co. (Dordrecht)

Pottasch, S. R., \& Acker, A. 1989, A\&A, 221, 123

Pottasch, S. R., \& Beintema, D. A. 1999, A\&A, 347, 974

Pottasch, S. R., \& Bernard-Salas, J. 2006, A\&A, 457, 189

Pottasch, S. R., Wesselius, P. R., Wu, C. C., et al. 1978, A\&A, 62, 95

Pottasch, S. R., Beintema, D. A., \& Feibelman, W. A. 2000, A\&A, 363, 767

Pottasch, S. R., Beintema, D. A., Bernard Salas, J., \& Feibelman, W. A. 2001, A\&A, 380, 684

Pottasch, S. R., Bernard-Salas, J., \& Roellig, T. L. 2007, A\&A, 471, 865

Preite-Martinez, A., \& Pottasch, S. R. 1983 A\&A, 126, 31

Seaton, M. J. 1979, MNRAS, 187, 73

Tinkler, C. M., \& Lamers, H. J. G. L. M. 2002, A\&A, 384, 987

Werner, M., Roellig, T. L., Low, F. J., et al. 2004, ApJS, 154, 1 